UCRHEP-T327

March 2002

\title{
New U(1) Gauge Symmetry of Quarks and Leptons
}

\author{
Ernest Ma \\ Physics Department, University of California, Riverside, California 92521, USA
}

\begin{abstract}
Instead of anchoring the seesaw mechanism with the conventional heavy righthanded neutrino singlet, a small Majorana neutrino mass may be obtained just as well with the addition of a heavy triplet of leptons per family to the minimal standard model of particle interactions. The resulting model is shown to have the remarkable property of accommodating a new U(1) symmetry which is anomaly-free and may thus be gauged. There are many possible phenomenological consequences of this proposal which may be already relevant in explaining one or two recent potential experimental discrepancies.
\end{abstract}


To obtain nonzero neutrino masses so as to explain the observed atmospheric [1] and solar [2] neutrino oscillations, the minimal standard model of particle interactions is often extended to include three neutral fermion singlets, often referred to as right-handed singlet neutrinos. If they have large Majorana masses, then the famous seesaw mechanism [3] allows the observed neutrinos to acquire naturally small Majorana masses. On the other hand, there are other equivalent ways [4, 5] to realize this effective dimension-five operator [6] for neutrino mass. For example, if we replace each neutral fermion singlet by a triplet: [5, 7]

$$
\Sigma=\left(\Sigma^{+}, \Sigma^{0}, \Sigma^{-}\right) \sim(1,3,0)
$$

under $S U(3)_{C} \times S U(2)_{L} \times U(1)_{Y}$, the seesaw mechanism works just as well.

It is well-known [8] that in the case of one additional right-handed singlet neutrino per family of quarks and leptons, it is possible to promote $B-L$ (baryon number - lepton number) from being a global $\mathrm{U}(1)$ symmetry to an $\mathrm{U}(1)$ gauge symmetry. Here I consider the case of one additional triplet of leptons per family, and prove the remarkable fact that a new $\mathrm{U}(1)$ symmetry exists which is anomaly-free and may thus be gauged. This discovery leads naturally to a number of possible interesting novel experimental consequences.

I assume $S U(3)_{C} \times S U(2)_{L} \times U(1)_{Y} \times U(1)_{X}$ as a possible extension of the standard model, under which each family of quarks and leptons transforms as follows:

$$
\begin{array}{ll}
(u, d)_{L} \sim\left(3,2,1 / 6 ; n_{1}\right), & u_{R} \sim\left(3,1,2 / 3 ; n_{2}\right), \quad d_{R} \sim\left(3,1,-1 / 3 ; n_{3}\right), \\
(\nu, e)_{L} \sim\left(1,2,-1 / 2 ; n_{4}\right), & e_{R} \sim\left(1,1,-1 ; n_{5}\right), \quad \Sigma_{R} \sim\left(1,3,0 ; n_{6}\right) .
\end{array}
$$

There are potentially four Higgs doublets $\left(\phi_{i}^{+}, \phi_{i}^{0}\right)$ with $U(1)_{X}$ charges $n_{1}-n_{3}, n_{2}-n_{1}$, $n_{4}-n_{5}$, and $n_{6}-n_{4}$. However, it will turn out that three of these four charges are identical, so this model only requires the minimum of two distinct Higgs doublets (to be compared with the minimum of one Higgs doublet in the standard model). To allow large Majorana 
masses for $\Sigma$, the Higgs singlet

$$
\chi^{0} \sim\left(1,1,0 ;-2 n_{6}\right)
$$

is also added.

To ensure the absence of the axial-vector anomaly [9], the following conditions are considered [10].

$$
\begin{gathered}
{[S U(3)]^{2} U(1)_{X}: 2 n_{1}-n_{2}-n_{3}=0} \\
{[S U(2)]^{2} U(1)_{X}: 3\left(\frac{1}{2}\right) n_{1}+\left(\frac{1}{2}\right) n_{4}-(2) n_{6}=0} \\
{\left[U(1)_{Y}\right]^{2} U(1)_{X}: 6\left(\frac{1}{6}\right)^{2} n_{1}-3\left(\frac{2}{3}\right)^{2} n_{2}-3\left(-\frac{1}{3}\right)^{2} n_{3}+2\left(-\frac{1}{2}\right)^{2} n_{4}-(-1)^{2} n_{5}=0} \\
U(1)_{Y}\left[U(1)_{X}\right]^{2}: 6\left(\frac{1}{6}\right) n_{1}^{2}-3\left(\frac{2}{3}\right) n_{2}^{2}-3\left(-\frac{1}{3}\right) n_{3}^{2}+2\left(-\frac{1}{2}\right) n_{4}^{2}-(-1) n_{5}^{2}=0 \\
{\left[U(1)_{X}\right]^{3}: 6 n_{1}^{3}-3 n_{2}^{3}-3 n_{3}^{3}+2 n_{4}^{3}-n_{5}^{3}-3 n_{6}^{3}=0 .}
\end{gathered}
$$

Furthermore, the absence of the mixed gravitational-gauge anomaly 11] requires the sum of $U(1)_{X}$ charges to vanish, i.e.

$$
U(1)_{X}: 6 n_{1}-3 n_{2}-3 n_{3}+2 n_{4}-n_{5}-3 n_{6}=0
$$

Since the number of $S U(2)_{L}$ doublets remains even (it is in fact unchanged), the global SU(2) chiral gauge anomaly [12] is absent automatically.

Equations (4), (6), and (7) do not involve $n_{6}$. Together they allow two solutions:

$$
\text { (I) } n_{4}=-3 n_{1}, \quad \text { (II) } n_{2}=\frac{1}{4}\left(7 n_{1}-3 n_{4}\right) \text {. }
$$

Using Eq. (5), solution (I) implies $n_{6}=0$, from which it can easily be seen that $U(1)_{X}$ is proportional to $U(1)_{Y}$. In other words, no new gauge symmetry has been discovered. 
Consider now solution (II). Using Eqs. (4) and (6), it implies

$$
n_{3}=\frac{1}{4}\left(n_{1}+3 n_{4}\right), \quad n_{5}=\frac{1}{4}\left(-9 n_{1}+5 n_{4}\right) .
$$

Equations (5), (8), and (9) are then all satisfied with

$$
n_{6}=\frac{1}{4}\left(3 n_{1}+n_{4}\right) .
$$

This is a remarkable and highly nontrivial result. In fact, it can be shown that the Casimir invariants of the $\mathrm{SU}(2)$ representations are such that the only solutions to the anomaly-free conditions are with either a singlet, i.e. $N_{R}$, or a triplet, i.e. $\Sigma_{R}$.

The $U(1)_{X}$ charges of the possible Higgs doublets are:

$$
n_{1}-n_{3}=n_{2}-n_{1}=n_{6}-n_{4}=\frac{3}{4}\left(n_{1}-n_{4}\right), \quad n_{4}-n_{5}=\frac{1}{4}\left(9 n_{1}-n_{4}\right),
$$

which means that two distinct Higgs doublets are sufficient for all possible Dirac fermion masses in this model. If $n_{4}=-3 n_{1}$ is chosen, then again $U(1)_{X}$ will be proportional to $U(1)_{Y}$. However, for $n_{4} \neq-3 n_{1}$, a new class of models is now possible with $U(1)_{X}$ as a genuinely new gauge symmetry.

To summarize, the quarks and leptons transform under $U(1)_{X}$ as follows:

$$
\begin{aligned}
& (u, d)_{L} \sim n_{1}, \quad u_{R} \sim \frac{1}{4}\left(7 n_{1}-3 n_{4}\right), \quad d_{R} \sim \frac{1}{4}\left(n_{1}+3 n_{4}\right), \\
& (\nu, e)_{L} \sim n_{4}, \quad e_{R} \sim \frac{1}{4}\left(-9 n_{1}+5 n_{4}\right), \quad \Sigma_{R} \sim \frac{1}{4}\left(3 n_{1}+n_{4}\right) .
\end{aligned}
$$

The above charge assignments do not correspond to any existing model of quark and lepton interactions. For example, if $n_{4}=n_{1}$ is assumed, then

$$
n_{1}=n_{2}=n_{3}=n_{4}=-n_{5}=n_{6},
$$

which means that $X$ couples vectorially to quarks, but its coupling to charged leptons is purely axial-vector. On the other hand, if $n_{4}=9 n_{1}$ is assumed, then

$$
n_{1}=1, \quad n_{2}=-5, \quad n_{3}=7, \quad n_{4}=9, \quad n_{5}=9, \quad n_{6}=3
$$


is a solution with $X$ coupling vectorially to charged leptons.

Consider $\nu q$ and $\bar{\nu} q$ deep inelastic scattering. It has recently been reported [13] by the $\mathrm{NuTeV}$ Collaboration that their measurement of the effective $\sin ^{2} \theta_{W}$, i.e. $0.2277 \pm 0.0013 \pm$ 0.0009 , is about $3 \sigma$ away from the standard-model prediction of $0.2227 \pm 0.00037$. In this model, the $X$ gauge boson also contributes with

$$
\begin{aligned}
J_{X}^{\mu}= & n_{1} \bar{u} \gamma^{\mu}\left(\frac{1-\gamma_{5}}{2}\right) u+n_{1} \bar{d} \gamma^{\mu}\left(\frac{1-\gamma_{5}}{2}\right) d \\
& +n_{2} \bar{u} \gamma^{\mu}\left(\frac{1+\gamma_{5}}{2}\right) u+n_{3} \bar{d} \gamma^{\mu}\left(\frac{1+\gamma_{5}}{2}\right) d+n_{4} \bar{\nu} \gamma^{\mu}\left(\frac{1-\gamma_{5}}{2}\right) \nu
\end{aligned}
$$

Assuming very small $X-Z$ mixing $(|\sin \theta|<<1)$, the effective neutrino-quark interactions are then given by

$$
\mathcal{H}_{\text {int }}=\frac{G_{F}}{\sqrt{2}} \bar{\nu} \gamma^{\mu}\left(1-\gamma_{5}\right) \nu\left[\epsilon_{L}^{q} \bar{q} \gamma_{\mu}\left(1-\gamma_{5}\right) q+\epsilon_{R}^{q} \bar{q} \gamma_{\mu}\left(1+\gamma_{5}\right) q\right],
$$

where

$$
\begin{aligned}
\epsilon_{L}^{u} & =(1-\xi)\left(\frac{1}{2}-\frac{2}{3} \sin ^{2} \theta_{W}\right)+n_{1} \zeta, \\
\epsilon_{L}^{d} & =(1-\xi)\left(-\frac{1}{2}+\frac{1}{3} \sin ^{2} \theta_{W}\right)+n_{1} \zeta, \\
\epsilon_{R}^{u} & =(1-\xi)\left(-\frac{2}{3} \sin ^{2} \theta_{W}\right)+n_{2} \zeta, \\
\epsilon_{R}^{d} & =(1-\xi)\left(\frac{1}{3} \sin ^{2} \theta_{W}\right)+n_{3} \zeta,
\end{aligned}
$$

with

$$
\begin{aligned}
\xi & =n_{4} \sin \theta\left(1-\frac{M_{Z}^{2}}{M_{X}^{2}}\right) \frac{g_{X}}{g_{Z}} \\
\zeta & =-\sin \theta\left(1-\frac{M_{Z}^{2}}{M_{X}^{2}}\right) \frac{g_{X}}{g_{Z}}+n_{4}\left(\frac{M_{Z}^{2}}{M_{X}^{2}}\right) \frac{g_{X}^{2}}{g_{Z}^{2}} .
\end{aligned}
$$

To account for the $\mathrm{NuTeV}$ result, i.e.

$$
\begin{aligned}
& \left(g_{L}^{e f f}\right)^{2}=\left(\epsilon_{L}^{u}\right)^{2}+\left(\epsilon_{L}^{d}\right)^{2}=0.3005 \pm 0.0014 \\
& \left(g_{R}^{e f f}\right)^{2}=\left(\epsilon_{R}^{u}\right)^{2}+\left(\epsilon_{R}^{d}\right)^{2}=0.0310 \pm 0.0011
\end{aligned}
$$


against the standard-model prediction, i.e.

$$
\left(g_{L}^{e f f}\right)_{S M}^{2}=0.3042, \quad\left(g_{R}^{e f f}\right)_{S M}^{2}=0.0301
$$

consider the following specific model as an illustration:

$$
n_{1}=0, \quad n_{2}=-\frac{3}{4}, \quad n_{3}=\frac{3}{4}, \quad n_{4}=1, \quad n_{5}=\frac{5}{4}, \quad n_{6}=\frac{1}{4} .
$$

The central values of the $\mathrm{NuTeV}$ measurements are then obtained with

$$
\xi=0.0061, \quad \zeta=0.0038
$$

implying that

$$
M_{X} \simeq 10\left(\frac{g_{X}}{g_{Z}}\right) M_{Z}, \quad \sin \theta \simeq 0.006\left(\frac{g_{Z}}{g_{X}}\right) .
$$

Whereas $M_{X} \sim 1 \mathrm{TeV}$ is certainly allowed by the present data, a smaller value of $\sin \theta$ is indicated by the precision measurements at the $Z$ pole. A comprehensive numerical analysis of this and the more general case of $n_{1} \neq 0$ will be given elsewhere 14 .

In atomic parity nonconservation, the dominant effect comes from the axial-vector coupling of the electron. In the model defined by Eq. (29), this is given by $\left(n_{4}-n_{5}\right) / 2=-1 / 8$; hence it is rather suppressed. Furthermore, the isoscalar vector coupling of the quarks in this model also vanishes. Therefore, the contribution of $X$ is essentially negligible and there should be no observable deviation from the prediction [15] of the standard model, in agreement with the most recent data [16].

Consider now the anomalous magnetic moment of the muon. A recent experimental result [17], after the latest theoretical corrections [18], gives its deviation from the standard model as

$$
\Delta a_{\mu}=2.5 \pm 1.6 \times 10^{-9}
$$

which is only an $1.6 \sigma$ effect. From the standpoint of the proposed $U(1)_{X}$ model, there are two possible contributions. One comes from the $X$ boson which has a vector coupling, i.e. 
$\left(n_{4}+n_{5}\right) / 2$, to the muon. However, if $M_{X} \sim 1 \mathrm{TeV}$, then this contribution is essentially negligible. The other comes from the extended Higgs sector of this model. In particular, the coupling of $\left(\nu_{\mu}, \mu\right)_{L}$ to $\Sigma_{R}$ through the Higgs doublet with $X$ charge $3\left(n_{1}-n_{4}\right) / 4$ provides two one-loop diagrams: one with $\Sigma^{-}$and $\bar{\phi}^{0}$ as intermediate states, the other with $\Sigma^{0}$ and $\phi^{-}$. If all these masses are equal, the former contributes with a coefficient of +2 and the latter with a coefficient of -1 to $\Delta a_{\mu}$. Assuming masses of order $200 \mathrm{GeV}$, it is thus possible to account for Eq. (32).

It is well-known that given its particle content, the minimal standard model does not allow for the unification of gauge couplings. The addition of $\Sigma_{R}$ in Eq. (2) does not change the situation. However, if gauge-coupling unification at $M_{U} \sim 10^{16} \mathrm{GeV}$ is desired, one simple possibility is to add three charged lepton singlets with only vector interactions, i.e. $E_{L, R} \sim$ $(1,1,-1 ; 0)$, as well as an $S U(3)$ octet of neutral colored fermions, i.e. $\psi_{L, R} \sim(8,1,0 ; 0)$. It is clear that this model would still be anomaly-free, but the evolution equations of the gauge couplings would now change, assuming of course that all new particles have masses of order $10^{2} \mathrm{GeV}$. Generically, the one-loop renormalization-group equations for the running of gauge couplings are given by

$$
\alpha_{i}^{-1}\left(M_{1}\right)=\alpha_{i}^{-1}\left(M_{2}\right)-\frac{b_{i}}{2 \pi} \ln \frac{M_{1}}{M_{2}}
$$

where $\alpha_{i} \equiv g_{i}^{2} / 4 \pi$ and $b_{i}$ are constants determined by the particle content contributing to $\alpha_{i}$. Here,

$$
\begin{aligned}
& b_{3}=-11+(3) \frac{4}{3}+4=-3 \\
& b_{2}=-\frac{22}{3}+(3) \frac{4}{3}+(2) \frac{1}{6}+(3) \frac{4}{3}=1 \\
& b_{Y}=(3) \frac{20}{9}+(2) \frac{1}{6}+(3) \frac{4}{3}=11 \\
& b_{X}=\frac{1}{12}\left(585 n_{1}^{2}-282 n_{1} n_{4}+177 n_{4}^{2}\right)=\left(40 \text { if } n_{4}=n_{1}=1\right) .
\end{aligned}
$$


Using the precision measurements [19]

$$
\alpha^{-1}\left(M_{Z}\right)=127.938 \pm 0.027, \quad \sin ^{2} \theta_{W}\left(M_{Z}\right)=0.23117 \pm 0.00016
$$

and the relationships

$$
\alpha_{2}^{-1}=\alpha^{-1} \sin ^{2} \theta_{W}, \quad \alpha_{1}^{-1}=\frac{3}{5} \alpha_{Y}^{-1}=\frac{3}{5} \alpha^{-1} \cos ^{2} \theta_{W}
$$

I find from Eqs. (33), (35), and (36) that

$$
\frac{M_{U}}{M_{Z}}=2.223 \times 10^{14}
$$

from which $\alpha_{3}^{-1}\left(M_{Z}\right)$ is predicted by Eqs. (33) and (34) to be 8.544, in good agreement with the experimental value [19] $\alpha_{3}\left(M_{Z}\right)=0.1192 \pm 0.0028$, i.e. $\alpha_{3}^{-1}=8.39(+0.20 /-0.19)$.

In the above, $U(1)_{Y}$ is normalized as in the standard model, but since the normalization of $U(1)_{X}$ is unknown, $g_{X}$ cannot be unified in analogy to $g_{Y}$. This also means that a two-loop analysis of $\alpha_{1,2,3}$ would not be possible because it would involve $g_{X}$. There is no obvious unification symmetry which includes the particle content of this model as an anomaly-free subset.

Instead of having one $\Sigma_{R}$ per family, consider the total of (A) one $\Sigma_{R}$, and (B) two $\Sigma_{R}$ 's for the three families of quarks and leptons. In either case, Eqs. (4), (6), and (7) are unchanged. Hence solution (II) of Eq. (10) is still valid, together with Eq. (11). The analog of Eq. (5) now implies

$$
\text { (A) } n_{6}=\frac{3}{4}\left(3 n_{1}+n_{4}\right), \quad \text { (B) } n_{6}=\frac{3}{8}\left(3 n_{1}+n_{4}\right)
$$

Whereas the analog of Eq. (9) is still automatically satisfied, that of Eq. (8) is not. On the other hand, if singlet $N_{R}$ 's are added with $X$ charges given as follows:

$$
\begin{aligned}
& \text { (A) : } n_{6}, n_{6},-\frac{1}{3} n_{6},-\frac{5}{3} n_{6}, \\
& \text { (B) : } n_{6}, \frac{2}{3} n_{6},-\frac{5}{3} n_{6},
\end{aligned}
$$


the analogs of both Eqs. (8) and (9) are again satisfied. Note that in Case (A), there are two singlets with $X$ charge $n_{6}$, and in Case (B), there is one such singlet. This means that the total number of triplets and singlets with $X$ charge $n_{6}$ is always three in each of the three models, thus allowing all three neutrinos to acquire small seesaw Majorana masses.

To conclude, three anomaly-free $U(1)_{X}$ models have been discovered. They are characterized by having fermions and Higgs bosons beyond those of the minimal standard model. In the simplest case, each family of quarks and leptons is supplemented by a triplet of leptons. In another case, i.e. (A), there is only one triplet for the three families, but there are four singlets with $X$ charges given by Eq. (42). In the third case, i.e. (B), there are two triplets and three singlets with $X$ charges given by Eq. (43). If $U(1)_{X}$ is a relevant gauge symmetry at or near the electroweak breaking scale, then it may already be implicated in some recent experimental data which show possible deviations from the standard model, such as the $\mathrm{NuTeV}$ result [13] and the muon $g-2$ measurement [17]. Of course, the main motivation for studying $U(1)_{X}$ is not predicated on these potential discrepancies, but rather on its fundamental theoretical appeal. Details of other possible phenomenological consequences will be discussed elsewhere [14].

This work was supported in part by the U. S. Department of Energy under Grant No. DEFG03-94ER40837. 


\section{References}

[1] S. Fukuda et al., Super-Kamiokande Collaboration, Phys. Rev. Lett. 85, 3999 (2000) and references therein.

[2] S. Fukuda et al., Super-Kamiokande Collaboration, Phys. Rev. Lett. 86, 5656 (2001) and references therein. See also Q. R. Ahmad et al., SNO Collaboration, Phys. Rev. Lett. 87, 071301 (2001).

[3] M. Gell-Mann, P. Ramond, and R. Slansky, in Supergravity, edited by P. van Nieuwenhuizen and D. Z. Freedman (North-Holland, Amsterdam, 1979), p. 315; T. Yanagida, in Proceedings of the Workshop on the Unified Theory and the Baryon Number in the Universe, edited by O. Sawada and A. Sugamoto (KEK, Tsukuba, Japan, 1979), p. 95;

R. N. Mohapatra and G. Senjanovic, Phys. Rev. Lett. 44, 912 (1980).

[4] E. Ma and U. Sarkar, Phys. Rev. Lett. 80, 5716 (1998).

[5] E. Ma, Phys. Rev. Lett. 81, 1171 (1998).

[6] S. Weinberg, Phys. Rev. Lett. 43, 1566 (1979).

[7] R. Foot, H. Lew, X.-G. He, and G. C. Joshi, Z. Phys. C44, 441 (1989).

[8] R. E. Marshak and R. N. Mohapatra, Phys. Lett. 91B, 222 (1980).

[9] S. L. Adler, Phys. Rev. 177, 2426 (1969); J. S. Bell and R. Jackiw, Nuovo Cimento A60, 47 (1969); W. A. Bardeen, Phys. Rev. 184, 1848 (1969).

[10] C. Q. Geng and R. E. Marshak, Phys. Rev. D39, 693 (1989); X.-G. He, G. C. Joshi, and R. R. Volkas, Phys. Rev. D41, 278 (1990). 
[11] R. Delbourgo and A. Salam, Phys. Lett. 40B, 381 (1972); T. Eguchi, and P. G. O. Freund, Phys. Rev. Lett. 37, 1251 (1976); L. Alvarez-Gaume and E. Witten, Nucl. Phys. B234, 269 (1984).

[12] E. Witten, Phys. Lett. 117B, 324 (1982).

[13] G. P. Zeller et al., NuTeV Collaboration, Phys. Rev. Lett. 88, 091802 (2002).

[14] E. Ma and D. P. Roy, in preparation.

[15] A. Derevianko, Phys. Rev. Lett. 85, 1618 (2000); M. G. Kozlov, S. G. Porsev, and I. I. Tupitsyn, Phys. Rev. Lett. 86, 3260 (2001); V. A. Duzba, C. Harabati, W. R. Johnson, and M. S. Safronova, Phys. Rev. A63, 044103 (2001).

[16] S. C. Bennett and C. E. Wieman, Phys. Rev. Lett. 82, 2484 (1999).

[17] H. N. Brown et al., Phys. Rev. Lett. 86, 2227 (2001).

[18] M. Knecht and A. Nyffeler, hep-ph/0111058; M. Knecht, A. Nyffeler, M. Perrottet, and E. de Rafael, Phys. Rev. Lett. 88, 071802 (2002); M. Hayakawa and T. Kinoshita, hepph/0112102; I. Blokland, A. Czarnecki, and K. Melnikov, Phys. Rev. Lett. 88, 071803 (2002).

[19] Particle Data Group, Eur. Phys. J. C15, 1 (2000). 